\title{
Selectively attending to natural scenes after alcohol consumption: An ERP analysis
}

\author{
Andrea De Cesarei ${ }^{a}$, Maurizio Codispoti ${ }^{\mathrm{a}, *}$, Harald T. Schupp ${ }^{\mathrm{b}}$, Luciano Stegagno ${ }^{\mathrm{c}}$ \\ a Department of Psychology, Universita' degli Studi di Bologna, Viale Berti Pichat, 5-40127 Bologna, Italy \\ ${ }^{\mathrm{b}}$ University of Konstanz, Department of General Psychology, Universitätsstraße, 10-D78457 Konstanz, Germany \\ ${ }^{\mathrm{c}}$ University of Padova, Department of General Psychology, Via Venezia, 8-35131 Padova, Germany
}

Received 25 January 2005; accepted 23 June 2005

\begin{abstract}
Alcohol effects on cognitive, emotional and behavioral processes have been linked to an impairment of attention. Because attention operates at the level of specific cognitive subsystems, recent studies demonstrated alcohol effects in specific post-perceptual processes such as response selection and working memory. Measuring event-related potentials, the present study focused on perceptual processes by utilizing a categorization task where participants had to decide whether briefly presented images contained an animal or not. Findings demonstrate an early differential ERP activity for target compared to non-target images, which was reduced after alcohol intoxication. Thus, alcohol intoxication had deleterious effects at the perceptual level of processing considered to reflect the interaction of top-down (category-related) and bottom-up (stimulus-driven) processes. In addition, post-perceptual processes were also impaired by alcohol intoxication.
\end{abstract}

(C) 2005 Published by Elsevier B.V.

Keywords: Alcohol; Attention; Motivation; ERPs; Natural scenes

It is widely known that alcohol deeply impacts behavior, cognitive processes and emotions. In several years of research, the mechanisms of alcohol action have been extensively studied, pointing out that the impairment of attentional functions plays a main role in determining alcohol effects (Steele and Josephs, 1988; Maylor and Rabbitt, 1993; Curtin et al., 2001). However, alcohol effects may also be delineated at the level of specific cognitive subsystems and processes (cf., Luck et al., 2000). For instance, recent studies provide strong evidence for selective alcohol impairments at post-perceptual stages of information processing (OscarBerman, 1987; Polich and Kok, 1995; Marinkovic et al., 2001; Bartholow et al., 2003). Pursuing the goal of examining the effects of alcohol in relation to specific cognitive subsystems, the present study examined the fundamental capability of stimulus categorization. The ability to categorize visual objects at the superordinate level is a crucial component of our everyday interaction with the environment,

* Corresponding author. Tel:: +39 051 2091836; fax: +39 051243086. E-mail address: maurizio.codispoti2@unibo.it (M. Codispoti). resulting from the interaction of perceptual analysis (bottomup) with semantic knowledge (top-down). As categorization proceeds in a sequence of stages, event-related brain potentials (ERPs) might index the effects of alcohol intoxication on perceptual and post-perceptual/evaluative stages of information processing.

\section{Alcohol and selective attention}

From a theoretical perspective, the adverse consequences of alcohol intoxication on disregulated behaviors, emotion and cognition have been primarily considered to reflect impairments in attention functions (Steele and Josephs, 1988; Maylor and Rabbitt, 1993; Curtin et al., 2001). Acknowledging the multi-faceted nature of attentional processes (e.g. Pashler, 1999), alcohol intoxication may be expressed as specific impairments in cognitive subsystems such as stimulus identification, working memory and response selection (Luck et al., 2000; Luck and Hillyard, 2000). Considered from this perspective, the effects of 
alcohol on attention are expected to depend on the nature of the stimuli and the task, and occur in specific subsystems, which may suffer from depletion of resources caused by intoxication. ERPs are particularly suited to examining the link between attention and alcohol at the level of specific cognitive subsystems (Luck and Hillyard, 2000). For instance, Curtin and Fairchild (2003) demonstrated that alcohol intoxication specifically impaired attentional processes at the response stage in a task where participants had to resolve conflicting responses. The disruption of higher cognitive processes may also underlie the effects of alcohol on emotion (Curtin et al., 2001). However, alcohol effects have been reported not only during later stages of information processing, but also during the perceptual processing stage (Jääskeläinen et al., 1996). Both in the auditory and visual modality, specific ERP components linked to perceptual processing were attenuated under alcohol intoxication (for an extensive review, see Jääskeläinen et al., 1996). The majority of selective attention studies implemented tasks relying on simple stimulus features (e.g. pure tones, shapes, colors, etc.), which allow a great degree of control against possible perceptual confounding factors. However, in alcohol research it is currently unknown whether complex scenes, similar to what people are likely to encounter in real life, are subject to the same influences observed in attention tasks using simple stimuli. This class of stimuli differs from geometric shapes not only for the greater complexity and ecological relevance, but also because of its intrinsic semantic content, which has to be analyzed in order to recognize the stimulus. Therefore, it seems the ideal candidate when investigating alcohol impairment on complex categorization, which relies on semantic knowledge. Building on previous research with natural complex stimuli, the present study addressed the question of whether alcohol intoxication has deleterious effects on the ability to rapidly categorize real-world natural scenes.

\section{High-level categorization of complex scenes}

In order to facilitate the organization of goal-directed behaviors, visual attention can be voluntarily oriented to detecting target stimuli. The detection of target stimuli requires the cognitive system to be tuned in order to preferentially elaborate certain objects or features. This tuning, resulting in enhanced behavioral speed and accuracy, modulates various stages of processing and is reflected in scalp potentials. In particular, it has been shown that ERP components, which have been linked, respectively, to sensory analysis and decision stages (Kok, 2001) are modulated by explicit attention to visual features.

Previous studies have shown that paying attention to a class of stimuli results in an anterior positivity in the time window between 180 and $300 \mathrm{~ms}$, and in a posterior negativity at about the same latency. These components have been variously labeled $\mathrm{P} 2 \mathrm{a} / \mathrm{N} 2 \mathrm{~b}$, frontal selection positivity/ selection negativity (Potts, 2004; Kenemans et al., 1993; Hillyard and Anllo-Vento, 1998) and are supposed to index the tuning by higher level neural structures on lower level areas in order to detect one class of targets. Evaluating the question of whether early perceptual activity may be modulated based on semantic information, recent studies showed that differential early processing for targets can also be observed following complex natural scenes (Thorpe et al., 1996; Fabre-Thorpe et al., 2001; VanRullen and Thorpe, 2001b). For instance, Thorpe et al. (1996) asked participants to categorize briefly presented natural scenes according to whether they contained an animal or not. An early differential ERP activity for the attended stimulus category compared to the distractor items was observed at frontal sensor sites around $150 \mathrm{~ms}$ post-stimulus (Thorpe et al., 1996; VanRullen and Thorpe, 2001b). More recent studies indicate that rapid picture categorization is not limited to natural objects (e.g. animals) but extends also to the artificial object category (e.g. means of transport) with similar response times and ERPs across both categories (VanRullen and Thorpe, 2001a,b). Taking these results together, rapid picture categorization may be considered to reflect the interaction between bottom-up processing in the visual brain (Delorme et al., 2004; Fabre-Thorpe, 2003; VanRullen and Thorpe, 2001b), and top-down modulations, tuning the perceptual system for target category images.

In a system perceptually biased towards responding to a target category, targets are preferentially processed also at post-perceptual stages. An ERP component often observed in target-detection paradigms is the parietal P3 (P3b). Across different models, there is general agreement that this component reflects more controlled and attentional processes, which take place after stimulus identification. In addition, while early components reflect modulation of processing within a sensor modality, the P3 is largely modalityindependent (although some evidence suggests different brain sources in different modalities, e.g. Johnson, 1989). The amplitude of the P3 is modulated by task requirements and difficulty, and is usually larger for targets (Kok, 2001).

Thus, two stages of processing in the attention to categories may be identified, one related to stimulus identification, and the other to subsequent post-perceptual processes. In this view, effects of alcohol may be investigated at different stages of processing, in order to clarify the specificity of alcohol action.

\section{The current study}

The main goal of the present study was to determine whether alcohol intoxication can hamper categorization of complex real-world images according to higher-level class membership. As in previous studies by Thorpe et al. (1996), participants had to categorize images according to whether or not they contained an animal. However, while in the 
studies of Thorpe et al. a Go/NoGo response was required, in the present study participants had to respond as to whether an animal was present in the image or not (Antal et al., 2000). Based on the view that specific cognitive subsystems operate at different stages (Luck et al., 2000; Luck and Hillyard, 2000) we distinguish between ERP components related to perceptual and post-perceptual processing (Kok, 2001). Specifically, we examined the hypothesis that the early differential ERP activity elicited by target compared to non-target stimuli is reduced when alcohol intoxication occurs. Alternatively, rapid categorization of natural images displays several characteristics, which suggest that this task can be performed at an optimal level regardless of the amount of attentional resources available. Assuming that the effect of alcohol is exerted through a reduction of attentional resources, it is possible that the processing in this kind of task is not modulated by alcohol intoxication. In addition to early, perceptual-related ERP components, we were interested in the assessment of the P3, the late post-perceptual ERP component. In line with previous findings (OscarBerman, 1987; Polich and Kok, 1995; Marinkovic et al., 2001; Bartholow et al., 2003), it was predicted that P3 amplitude is reduced under alcohol intoxication compared to the placebo control condition. Furthermore, it was predicted that alcohol intoxication also has an impact on overt behavior responses as reflected by slower reaction times and increased error rates.

A second goal of this study was to investigate the effects of an increased motivational state with regard to categorization. Thus, participants received in some blocks of the task a monetary reward. Giving participants an incentive has been demonstrated to be an effective way to enhance the amount of attention invested in the task as measured by behavioral responses (Locke et al., 1981) and ERPs (Johnson, 1988) and in some conditions to counteract alcohol effects (Fillmore and Vogel-Sprott, 2000). The main interest was in determining whether motivation is able to reduce the deleterious effects due to alcohol intoxication either at the level of perceptual or post-perceptual processing or at both levels.

\section{Method}

\subsection{Participants}

Seventeen people (nine females) took part in the present study. Participants were between the ages of 21 and 28 years $(M=24.35)$. None of the participants had any condition contraindicating alcohol intake. Participants were recruited from a database of potential research participants who responded to a questionnaire on drinking habits.

\subsection{Alcohol use}

In a questionnaire conducted before the first session, participants were requested to indicate the amount of alcohol usually consumed during the week and the weekend, and at different moments of the day (morning, lunch, afternoon, dinner, night, party occasions). Types of alcohol included beer, wine, liquor and aperitifs. The estimated amount of each beverage was weighted for the alcohol content and added up in order to obtain a single measure of alcohol habits. All participants had previous experience with the administered dose of alcohol, and none of them showed extreme values that could be indicative of substance abuse.

\subsection{Procedure}

\subsubsection{Consent}

Participants were informed of the time requirements of the study and of the reward and category manipulation. They were not informed that alcohol and placebo intake was required in separate sessions. At the beginning of each session participants signed an informed consent form.

\subsubsection{Design}

Each participant performed a natural categorization task (animal versus non-animal) in two sessions, with beverage (alcohol versus placebo), and reward (with versus without) as independent variables. The beverage was manipulated across sessions. Thus, half the participants had an alcoholic beverage in the first session and a non-alcoholic placebo beverage in the second session, while the order was reversed for the other half of the participants. Within each session, the task was performed in four blocks of 300 trials each, varying the reward across blocks. More specifically, half of the participants performed the first two blocks of the categorization task receiving a monetary reward and the last two blocks without reward. The order of blocks with reward was reversed in the second session for each participant, and was balanced across all participants. Both sessions began at the same time of day (11:00 a.m.) and were separated by a week.

\subsubsection{Beverage manipulation}

Participants had either an alcoholic or non-alcoholic placebo beverage in separate sessions. The alcoholic beverage consisted of one part of pure ethyl alcohol mixed with four parts of fruit juice. The alcohol content was adapted to each participant's weight and sex according to the formula $0.80 \mathrm{~mL} / \mathrm{kg}$ for men and $0.70 \mathrm{~mL} / \mathrm{kg}$ for women. Replacing the pure ethyl alcohol with non-alcoholic rum essence, the non-alcoholic placebo beverage was prepared otherwise identically to the alcoholic beverage. The rum essence was chosen in order to mimic the smell and taste of the alcoholic beverage. Participants received the drink at the beginning of each session, after signing the informed consent form, and were allowed to consume the beverage in $15 \mathrm{~min}$ followed by an absorption period of $30 \mathrm{~min}$. 


\subsubsection{Reward manipulation}

Delivery of a monetary reward (with versus without) was manipulated in separate task blocks. In reward blocks, participants received a monetary reward $(\phi 10)$ for each correct response, regardless of the speed of the response time. During reward and non-reward blocks, participants received feedback as to whether their response was correct or not. In addition, during reward trials, participants were informed of the total sum of money they had amassed. Participants were informed that the maximum amount of money they could receive was $€ 120$. Showing consistency with the high accuracy achieved in this task in previous studies, participants received on average $€ 114$.

\subsubsection{Categorization task}

The images were 2400 real-world pictures, taken from widely available commercial databases. Half of the stimuli contained animals of various types (fish, mammals, reptiles, insects, birds), while the rest contained pictures of people, houses, landscapes, objects and vegetation. The stimulus set was divided into eight presentation blocks, the order of which was balanced across participants and conditions. In all blocks animals were treated as targets, while all other stimuli were distractors with the same probability of occurrence. Each image was presented to participants only once, in order to prevent learning effects.

Within each session, participants performed four blocks of the categorization task with each block consisting of 300 trials. Within each block, participants were shown target and non-target stimuli with a probability of $50 \%$. Each picture was shown for $24 \mathrm{~ms}$, followed by a black screen for $1000 \mathrm{~ms}$ while participants indicated their response using a two-button keypad. Afterwards, feedback was presented to participants on their performance (and the money status in reward blocks) for $2000 \mathrm{~ms}$. The intertrial interval lasted on average $500 \mathrm{~ms}$.

\subsection{Electroencephalographic recording}

The electroencephalogram (EEG) was recorded from Fz, $\mathrm{Cz}$ and $\mathrm{Pz}$ according to the international 10-20 system using a Grass Model 15 amplifier and VPM data acquisition software (Cook, 1997). Scalp impedance for each sensor was kept below $10 \mathrm{k} \Omega$. All channels were referenced to the right mastoid. The EEG was recorded continuously with a sampling rate of $1000 \mathrm{~Hz}$ and on-line bandpass filtered from 0.01 to $100 \mathrm{~Hz}$. Vertical and horizontal eye movements were recorded using $\mathrm{Ag} / \mathrm{AgCl}$ electrodes in the same frequency range as the EEG. Vertical eye movements were recorded from one electrode placed above the right eyebrow and one placed $1 \mathrm{~cm}$ below the right eye. Horizontal eye movements were recorded from two electrodes placed $1 \mathrm{~cm}$ to the sides of the eyes. In the off-line analysis, continuous EEG data were low-pass filtered at $30 \mathrm{~Hz}$ using digital filtering before stimulus synchronized epochs were extracted from $100 \mathrm{~ms}$ before picture onset until $700 \mathrm{~ms}$ after picture onset. All individual trials with EEG or EOG activity above $\pm 150 \mu \mathrm{V}$ were excluded from further analysis. To correct for vertical and horizontal ocular artifacts, an eye movement artifact correction (Gratton et al., 1983; Miller et al., 1988) was applied to single trial EEG epochs. Finally, separate average waveforms were calculated separately for each picture category (target and non-target), beverage condition (alcohol and placebo), reward (with and without) and electrode site, referenced to a $100 \mathrm{~ms}$ baseline preceding picture onset.

\subsection{Dependent measures}

\subsubsection{Blood alcohol level}

In order to obtain an estimate of the blood alcohol level (BAL), samples of breath were recorded on participants' arrival and 10, 20 and 30 min after beverage consumption using a Syen Elettronica ET-CM ethylometer. While performing the categorization task, samples of breath were collected between the second and third task blocks and at the end of the session (dotted lines in Fig. 1). Due to technical reasons, it was not possible to collect samples of breath from five participants.

\subsubsection{Behavioral responses}

Behavioral responses were acquired using PST Software E-Prime $^{\circledR}$ (Psychology Software Tools, 1999). Accuracy and response time (in milliseconds) were collected for both correct and incorrect trials. Only response times of correct trials were analyzed.

\subsubsection{Event-related potentials}

Analysis of the ERP waveform focused on the early ERP activity and on the P3. Following international guidelines and previous studies (Picton et al., 2000; Jääskeläinen et al., 1998), ERP components were scored where their amplitude was maximal. Consistent with the literature, the early effect

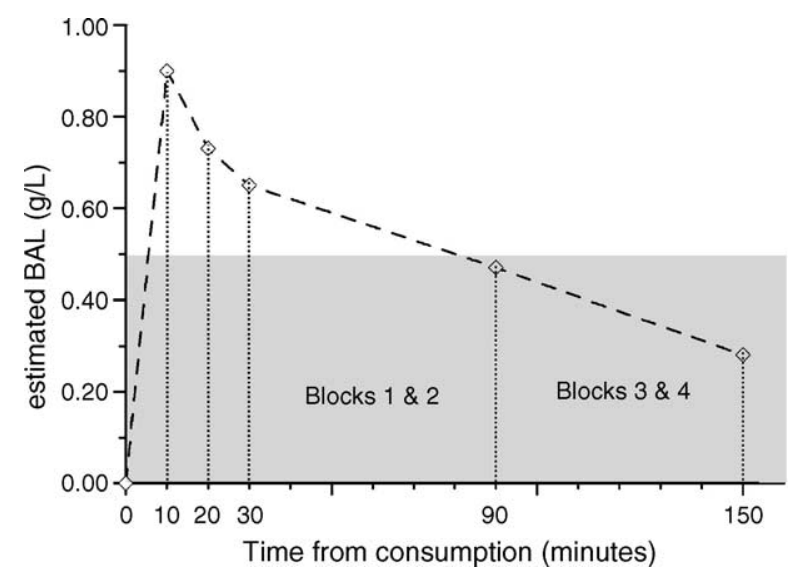

Fig. 1. Estimation of the BAL during the alcohol session. Grey zone represents the legal alcohol limit for driving in Italy. Dotted lines represent times of measurements. 

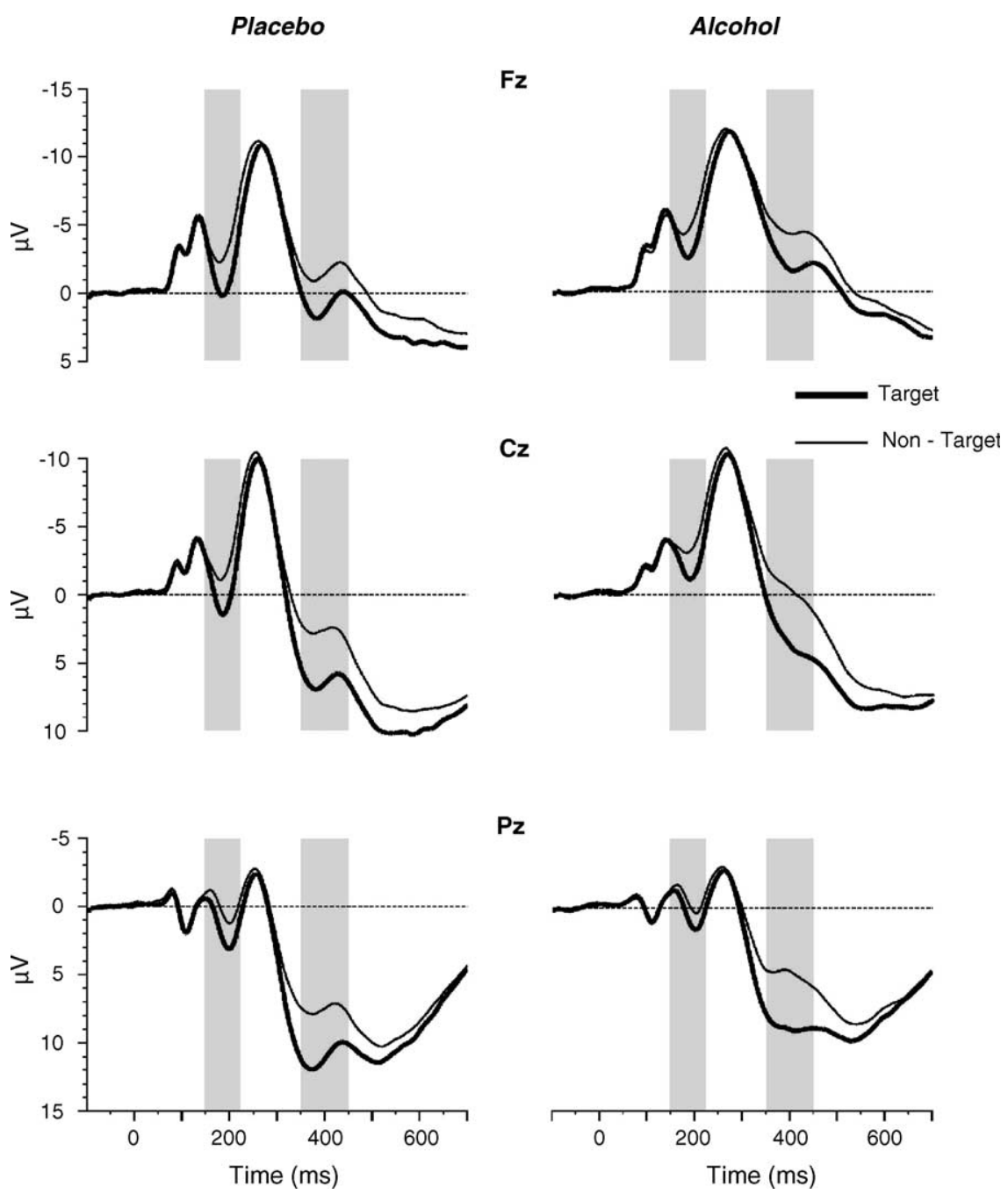

Fig. 2. ERPs for all sites, by Beverage and condition. Statistical analysis was conducted on the manually detected peak of the components highlighted in grey.

was examined at the Fz site (cf., Thorpe et al., 1996; VanRullen and Thorpe, 2001b), while the P3 analysis focused on Pz (cf., Johnson, 1988; Batty and Taylor, 2002). Amplitudes were scored by manually identifying the peak amplitude $^{1}$ of the ERP in the time window lasting from 150 to $220 \mathrm{~ms}$ after stimulus onset and from 350 to $450 \mathrm{~ms}$ (cf., Figs. 2 and 3).

\subsubsection{Other dependent measures}

In order to obtain a subjective estimation of beverage characteristics, participants were requested to evaluate the beverage on a scale of $0-10$ according to its pleasantness and alcohol content. Ratings were collected $30 \mathrm{~min}$ after beverage consumption, both in the alcohol and in the placebo session.

\footnotetext{
${ }^{1}$ Analysis of latency data indicated no significant interactions involving the factors Beverage or Reward, and are omitted for brevity.
}

\section{Results}

\subsection{Beverage administration manipulation check}

Estimated blood alcohol level during the experiment is shown in Fig. 1. All participants revealed an estimated BAL score of zero upon arrival at the laboratory. Ten minutes after alcohol ingestion the estimated BAL level reached the maximum $(0.90 \mathrm{~g} / \mathrm{L})$, and then decreased gradually to a value of $0.73 \mathrm{~g} / \mathrm{L}, 20 \mathrm{~min}$ after consumption. Measures of estimated BAL before, in the middle and after the task showed, respectively, values of $0.65 \mathrm{~g} / \mathrm{L}$ (S.D. $=0.15$ ), $0.47 \mathrm{~g} / \mathrm{L}$ (S.D. = 0.11) and 0.28 g/L (S.D. = 0.14). Relevant to the analysis of behavioral responses and ERPs, a Time (pre-task, mid-task, post-task) analysis of variance (ANOVA) was performed on the BAL scores before Block 1, between Blocks 2 and 3, and after Block 4, for sessions in which participants received alcoholic beverages. A significant main effect of Time was observed, $F(2,11)=40.3$, $p<0.001, \eta^{2}=0.89$. As expected, pairwise comparisons 

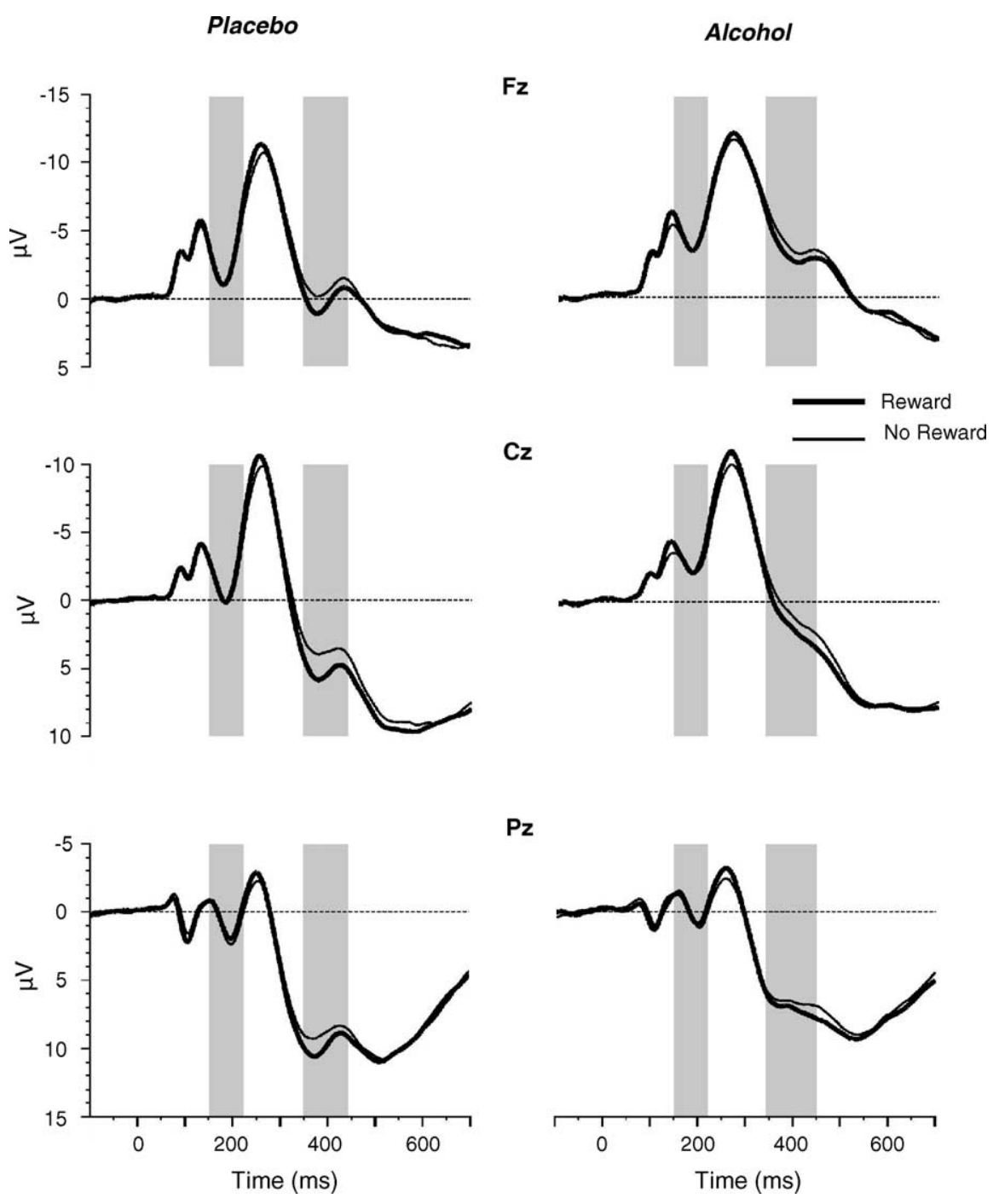

Fig. 3. Combined effect of Alcohol and Reward on ERPs, at all sites.

indicated that all contrasts were significant, $t_{\mathrm{s}}>5.67$, $p_{\mathrm{s}}<0.001$. Thus, all four blocks were performed during the descending limb of the BAL curve.

\subsection{Subjective reports}

In both sessions, participants were asked to evaluate the beverage according to its pleasantness and alcohol content. Results showed that the alcoholic beverage was rated more pleasant than the placebo (5.12 versus 3.47), $F(1,16)=4.62$, $p<0.05, \eta^{2}=0.22$. More important, the alcoholic beverage was perceived significantly more alcoholic than the placebo (7.29 versus 2.59), $F(1,16)=68.82, p<0.001, \eta^{2}=0.81$. Interestingly, despite the placebo beverage contained no ethanol, only three participants rated its alcohol content as zero. Furthermore, there was no order effect between participants who consumed in the first session the alcoholic beverage and in the second the placebo or vice versa, $F(1$, 16) $<1 \mathrm{~ns}$.

\subsection{Behavioral effects}

Accuracy and response time data were to separate repeated measures ANOVAs including the factors Beverage (alcohol versus placebo), Reward (with versus without) and Stimulus Category (target versus non-target). With regard to the response time data (see Table 1), a significant main effect

Table 1

Accuracy and reaction times according to Beverage, Reward and Stimulus Category

\begin{tabular}{llllll}
\hline Condition & \multicolumn{2}{l}{ Reaction time $(\mathrm{ms})$} & & \multicolumn{2}{l}{ Accuracy $(\%)$} \\
& Target & Non-target & & Target & Non-target \\
\hline Placebo & & & & & \\
$\quad$ Reward & $502(52.30)$ & $537(51.71)$ & & $97.37(1.53)$ & $96.45(2.90)$ \\
$\quad$ No reward & $525(55.38)$ & $551(58.05)$ & & $96.02(2.16)$ & $95.63(2.90)$ \\
Alcohol & & & & \\
$\quad$ Reward & $526(57.64)$ & $554(51.34)$ & & $93.00(9.41)$ & $93.76(5.33)$ \\
$\quad$ No reward & $534(48.64)$ & $557(44.49)$ & & $94.39(3.56)$ & $94.27(2.96)$ \\
\hline
\end{tabular}

Values are mean (S.D.). 
for the Category factor was observed, indicating faster response times to target compared to non-target stimuli, $F(1$, 16) $=59.1, p<0.001, \eta^{2}=0.79$. Furthermore, a significant main effect of the Beverage factor revealed slower responses in the alcohol compared to the placebo condition, $F(1$, 16) $=5.2, \quad p<0.05, \eta^{2}=0.24$. The analysis of data regarding accuracy revealed a significant main effect of the Beverage factor, $F(1,16)=8.4, p<0.05, \eta^{2}=0.34$, indicating slightly more errors after consumption of alcoholic compared to placebo beverages (see Table 1). No other main effects or higher-order interactions were significant in these analyses.

\subsection{ERP measures}

The main findings regarding the effects of Beverage, Reward and Stimulus Category are summarized in Figs. 2 and 3. As illustrated in Fig. 2, present results replicate previous findings in that target pictures were accompanied by enhanced early activity and P3 for targets compared to non-target pictures (Batty and Taylor, 2002; Codispoti et al., submitted for publication). Furthermore, although under alcohol intoxication a modulation of both early activity and P3 was observed compared to the placebo condition, alcohol differentially affected these two components. At the perceptual level of processing, alcohol effects appeared to be most prominent during the processing of target pictures. More specifically, the reduced amplitude of the early differential activity after alcohol consumption appeared to be more pronounced for target images. These results contrast with the post-perceptual processing indexed by the P3 component. Alcohol consumption was shown to be accompanied by generally smaller P3 components, similarly pronounced for target and non-target pictures. As shown in Fig. 3, monetary reward, presumably boosting task motivation, failed to modulate either the early differential activity or the $\mathrm{P} 3$ amplitude.

These descriptive findings were supported by the statistical analyses of the early ERP activity and P3, which were separately submitted to repeated measures ANOVAs containing factors Beverage (alcohol versus placebo), Reward (with versus without) and Stimulus Category (target versus non-target). ${ }^{2}$

\subsection{Early activity}

Replicating previous results, target compared to nontarget pictures elicited a more pronounced early ERP activity, Stimulus Category $F(1,16)=67.41, p<0.001$, $\eta^{2}=0.81$. Furthermore, alcohol consumption compared to

\footnotetext{
${ }^{2}$ In order to characterize present results with regard to individual differences, we performed a bivariate correlation between alcohol habit, maximum estimated BAL, and differences alcohol-placebo in reaction times, early component amplitude, P3 amplitude and early differential activity. Results failed to show any significant correlation.
}

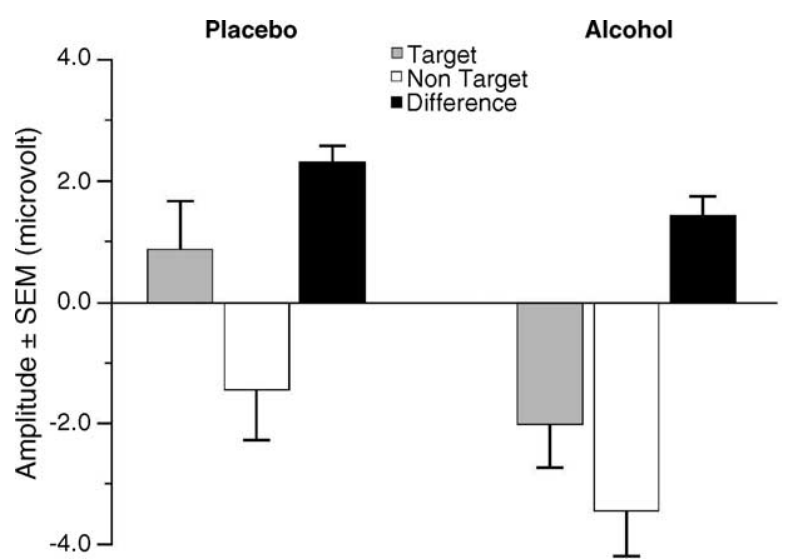

Fig. 4. Bar graph illustrating the reduction of the early $(150-220 \mathrm{~ms})$ activity following alcohol consumption. Black bars represent the difference targets - non-targets.

placebo beverages was reflected in a general reduction of the ERP peak, Beverage $F(1,16)=22.14, p<0.01$, $\eta^{2}=0.58$. In addition, the interaction Category $\times$ Beverage Beverage was significant, $F(1,16)=5.87, p<0.05$, $\eta^{2}=0.27$, indicating that the early ERP activity for target compared to non-target pictures was less pronounced after alcohol compared to placebo consumption. ${ }^{3}$ Decomposing the interaction into separate post hoc tests revealed that, although alcohol significantly reduced the ERP peak compared to placebo beverage, this effect was more pronounced for target rather than non-target picture processing, $t(16)=5.18, \quad p<0.001$ and $t(16)=3.7$, $p<0.01$, respectively (see Fig. 4).

No main effects or interactions involving factor Reward were observed in these analyses, $F_{\mathrm{s}}(1,16)<1 \mathrm{~ns}$.

\section{6. $P 3$}

Replicating previous findings, P3 amplitude was augmented for target compared to non-target images, $F(1$, $16)=146.6, p<0.001, \eta^{2}=0.9$. A significant main effect of Beverage was observed $F(1,16)=9.45, p<.01, \eta^{2}=.37$, indicating that the amplitude of the P3 was significantly attenuated for the alcohol compared to the placebo beverage condition. However, the interaction Beverage $\times$ Stimulus Category was not significant, $F(1,16)<1 \mathrm{~ns}$. Finally, the enhancement of the P3 component in rewarded blocks approached but did not reach conventional significance $F(1$, 16) $=3.89, p=0.066, \eta^{2}=0.2$.

No other higher-order interactions involving Reward, Alcohol and Stimulus Category were observed.

\footnotetext{
${ }^{3}$ In order to confirm the reduction of the early target discrimination, the early differential activity was also scored as the average of the difference wave target - non-target in the $150-300 \mathrm{~ms}$ time window. These data were submitted to a one-way ANOVA including factor Alcohol. Results of the ANOVA confirmed that following alcohol consumption the early differential activity is significantly reduced compared to the placebo session, $F(1$, 16) $=5.25, p<.05, \eta^{2}=.25$.
} 


\subsection{Control analyses: the effects of alcohol across time}

As mentioned at the beginning of Section 5, all four blocks were performed during the descending limb of the BAL curve. According to some evidence (Hurst and Bagley, 1972; Jones, 1973; Jones and Vega, 1972; but see Pihl et al., 2003, for a different conclusion), alcohol effects would be less pronounced during the descending rather than the ascending limb of the BAL curve. Since the ascending part of the BAL curve lasted in the present study only $10 \mathrm{~min}$, we are not able to address this issue. Nonetheless, it is worth pointing out that the effects were observed on the descending limb of the BAL curve. Since the effects of alcohol might have varied across time, we scored behavioral responses and ERP components for the first two blocks separately from the second two blocks. Submitting the behavioral responses to a three-way ANOVA including factors Beverage, Stimulus Category and Time (Blocks 1 and 2 versus Blocks 3 and 4 ) revealed no interaction of factor Time with Beverage and Stimulus Category on any of the considered variables, $F_{\mathrm{s}}(1,16)<1 \mathrm{~ns}$.

\section{Discussion}

The present study had the primary goal of examining the effects of alcohol intoxication on the fundamental process of categorization, which is a prerequisite for adaptive behaviors and efficient responses. With this objective, participants performed the categorization task in separate sessions consuming either alcoholic or placebo beverages. The results regarding the placebo control condition successfully replicated previous findings (Thorpe et al., 1996; VanRullen and Thorpe, 2001a,b). Interestingly, even though behavioral responses following alcohol consumption were significantly worse compared to the placebo condition, participants nonetheless demonstrated a rather high accuracy and only slightly delayed response times in categorization after alcohol intake (cf., Table 1). Furthermore, target compared to non-target pictures were associated with early and late ERP differences indexing the perceptual and post-perceptual stages of information processing. In line with the central tenet of identifying specific cognitive subsystems influenced by alcohol (cf., Luck et al., 2000), the present study observed alcohol impairments at the level of perceptual and postperceptual processing stages when participants classified natural images as to whether they contained an animal or not. Three findings are particularly noteworthy: (1) alcohol effects were demonstrated for early differential ERP activity, and particularly affected the processing of target pictures. (2) Although alcohol impaired both perceptual and postperceptual processing, the specific modulations induced by alcohol intoxication differed for both processing stages. (3) Monetary reward did not modulate the early differential ERP activity and, furthermore, did not alleviate the detrimental effects of alcohol consumption. Taken together, the present study demonstrated the impairments of alcohol on specific cognitive subsystems in a fundamental task, categorizing natural objects in the environment into superordinate groups.

The most interesting finding of the present study was the alcohol-induced reduction of the early ERP activity differentiating target from non-target images. Specifically, more prominent alcohol effects were observed during the processing of target items than during the processing of the non-target items. According to the presented results, alcohol consumption may impact selective processes prompting the visual system to respond to a specific category.

A number of findings link the observed component to selective attention. In target detection tasks, a family of ERP components related to target selection (Potts, 2004) is observed in the time window between 180 and $300 \mathrm{~ms}$. In particular, at frontal sites the P2a, P3f and Frontal Selection Positivity are enhanced for target stimuli (Kenemans et al., 1993; Potts and Tucker, 2001; Makeig et al., 1999). Interestingly, these components reflect a task-dependent mechanism as they are enhanced for target stimuli (Kenemans et al., 1993; Michie et al., 1999) regardless of the kind of response required (Potts, 2004). For instance, VanRullen and Thorpe (2001b) demonstrated an early differential ERP activity comparing the ERP waveform to identical stimulus categories (animals and vehicles) when they were taskrelevant compared to task-irrelevant. Accordingly, the early differential ERP activity observed for target compared to non-target images is considered to reflect not only bottomup stimulus-driven processing, but also top-down processes, presumably related to the representation of categorydefining stimulus characteristics (VanRullen and Thorpe, 2001b). Considered from this perspective, alcohol consumption may impact bottom-up and/or top-down processes.

Bottom-up processing in the visual ventral pathway, considered particularly relevant for object recognition, involves a hierarchical cascade of cortical regions accomplishing increasingly complex processing. Assuming that alcohol effects were predominantly impacting bottom-up processing, one might expect alcohol effects to be expressed similarly for the visual processing of all images irrespective of task status. Consistent with this hypothesis, the present data indicate a general reduction of the early perceptual component in the ERP waveform. A general decrease in arousal level might have contributed to this unspecific reduction. However, this reasoning is unlikely to explain the observed interaction of Stimulus Category and Beverage, indicating that the impact of alcohol on the early perceptual ERP component is most prominent for the processing of target images. It therefore appears likely that alcohol effects also impact top-down processes.

Recent theories specify how top-down influences may shape processing in the ventral visual pathway (Itti and Koch, 2001; Bar, 2003). Specifically, it has been proposed that feature-specific enhancement of processing in the visual areas for specific categories is implemented via prefrontal connections to visual-associative cortical regions. Support 
for the hypothesis that the prefrontal cortex organizes topdown effects on visual processing is derived from the anatomical connectivity of prefrontal regions (Selemon and Goldman-Rakic, 1988) and single-cell recordings showing early prefrontal activations ascending presumably from early visual areas (cf., Bar, 2003). Recently, a fMRI connectivity analysis provided experimental evidence for the involvement of the prefrontal cortex in regulating the activity in the visual cortical areas (Büchel and Friston, 1997). According to these findings, the early differential ERP activity may be considered to reflect a top-down mediated gain mechanism facilitating the processing of images containing category-defining stimulus features, specifically impaired by alcohol consumption.

The selective processing of target stimuli extends into later stages of stimulus processing. Specifically, the P3 amplitude is considered to reflect the amount of attention devoted to stimulus analysis and may represent working memory processes (Donchin and Coles, 1988). Alcohol consumption was observed to reduce P3 amplitudes to both target and non-target images compared to the placebo condition and, in contrast to the effects for the early ERP component, appeared not particularly prominent for target stimulus processing. Since it does not appear specific to target or non-target stimuli, this result could be related to a number of unspecific factors including lowered arousal or reduction of processing resources. These findings provide further support to differentiate alcohol effects at specific stages of cognitive subsystems, where most taxed stages (in this case, early perceptual) are the most heavily affected by alcohol consumption. Accordingly, the finding of unspecific reductions of the $\mathrm{P} 3$ component might be limited to the present context, given the relatively low demands of this task. It is likely that putting task demands on working memory capacity might reveal specific alcohol impairments to target stimuli. Support for this hypothesis is derived from a study by Curtin et al. (2001), which demonstrated specific P3 reductions to target stimuli in a dual-task situation following alcohol consumption.

The present study also evaluated the effects of alcohol to detect animals in natural contexts with respect to a condition where motivational state was either high or low. It was expected that in the rewarded condition alcohol effects would have been attenuated. This was not found, neither at the level of the early perceptual or late processing, nor at the level of behavioral performance. These results contrast with other studies demonstrating that motivation and expectancies were effective in counteracting negative effects of alcohol consumption (Myrsten et al., 1979; Fillmore and Vogel-Sprott, 2000). It is likely that, due to the high expertise achieved by participants in this kind of task, processing stages which would benefit more from deployment of attentional resources are not heavily taxed by this kind of task and, therefore, participants are not advantaged by an enhanced motivational state. In line with this reasoning, accuracy in the present study was notably high, which suggests that the task could be performed with low deployment of attentional resources, leading to a ceiling effect. Therefore, more studies varying attentional load are needed to provide a complete picture of the interaction of motivation and alcohol at the level of specific subsystems. However, though this result can be attributed to the low demands in this kind of task, one has to consider other alternative explanations. First of all, the lack of statistical significance may be due to insufficient statistical power. However, the strong effects observed with regard to the factors Beverage and Category cast some doubt whether increasing statistical power would have great effects in this type of task. Another alternative explanation for the results might derive from our choice to emphasize accuracy over speed. This may have led participants to adopt a strategy of slow responding, in order to achieve better results. However, following this reasoning one should expect reaction times to be slower than in comparable studies, and slower in the nonrewarded condition than in the rewarded one. Instead, RTs in the present study were only slightly slower than in the original investigation by Thorpe et al. (1996), who used a go/ no go paradigm, and even faster than Antal et al. (2000), who adopted the same two-choice task as in the present study. Similarly, there was no significant difference between RTs in rewarded and non-rewarded condition. Taken together, while the task of detecting animals in natural contexts was pronouncedly affected by alcohol intoxication, comparably little effects were observed when the task was performed under low or high motivation achieved by providing substantial monetary reward.

\subsection{Summary}

Many of the deleterious effects of alcohol intoxication may be directly or indirectly linked to impairments of attentional processes (Steele and Josephs, 1988; Maylor and Rabbitt, 1993; Curtin et al., 2001). In characterizing attentional impairments, research based on behavioral data derived the view of unspecific alcohol effects on attention rather than specific stages of information processing (cf., Maylor and Rabbitt, 1993). However, building upon recent advances in neuroscience, recent researches utilized ERP recordings to assess alcohol effects on specific stages of processing. This endeavor was based on the assumption that attention operates at the level of specific cognitive subsystems, which can only be observed when the subsystem in question is taxed by the particular task characteristics (Luck and Hillyard, 2000). Accordingly, recent findings seem to indicate that alcohol effects appear most pronounced in those cognitive subsystems targeted by the task. For instance, investigating stroop-like response interferences, Curtin et al. (2003) observed that the P3 component was not modulated by alcohol, but that alcohol intoxication reduced response-related ERP components. Similarly, in the present study, processing of target images was more hampered at the perceptual rather than the post- 
perceptual stage of information processing. These studies provide strong support for the hypothesis that alcohol intoxication affects attentional processes at the level of specific subsystems. However, each cognitive subsystem reveals attentional modulation when overloaded. Consequently, one might also expect that alcohol effects might be possible in any of the various cognitive subsystems. Both the level of alcohol intoxication and the demands on specific subsystems imposed by the chosen task play a role in determining whether alcohol intoxication impairs specific cognitive subsystems or not. This perspective entails many outstanding speculations and questions, which need to be addressed by future research regarding the relationship between alcohol intoxication, attention and cognitive subsystems.

\section{Acknowledgements}

This experiment was conducted in the Psychophysiology Lab at the University of Padova, Italy. We wish to thank Sara Salvatelli for her contribution to data collection, Pietro Scatturin for his skillful help in developing data-analysis software and Jasper Brener for helpful comments on a previous draft of this manuscript. We also thank all the people who volunteered to participate in the present study.

\section{References}

Antal, A., Keri, S., Kovacs, G., Janka, Z., Benedek, G., 2000. Early and late components of visual categorization: an event-related potential study. Brain Research/Cognitive Brain Research 9 (1), 117-119.

Bar, M., 2003. A cortical mechanism for triggering top-down facilitation in visual object recognition. Journal of Cognitive Neuroscience 15 (4), $600-609$.

Bartholow, B.D., Pearson, M., Sher, K.J., Wieman, L.C., Fabiani, M., Gratton, G., 2003. Effects of alcohol consumption and alcohol susceptibility on cognition: a psychophysiological examination. Biological Psychology 64, 167-190.

Batty, M., Taylor, M.J., 2002. Visual categorization during childhood: an ERP study. Psychophysiology 39 (4), 482-490.

Büchel, C., Friston, K.J., 1997. Modulation of connectivity in visual pathways by attention: cortical interactions evaluated with structural equation modelling and fMRI. Cerebral Cortex 7 (8), 768-778.

Codispoti, M., Ferrari, V., Junghoefer, M., Schupp, H.T. Scalp topography of the visual processing stream during the categorization of natural scenes, submitted for publication.

Cook III, E.W., 1997. VPM Reference Manual. Birmingham, Alabama.

Curtin, J.J., Fairchild, B.A., 2003. Alcohol and cognitive control: implications for regulation of behavior during response conflict. Journal of Abnormal Psychology 112 (3), 424-436.

Curtin, J.J., Patrick, C.J., Lang, A.R., Cacioppo, J.T., Birbaumer, N., 2001. Alcohol affects emotion through cognition. Psychological Science 12 (6), 527-531.

Delorme, A., Rousselet, G.A., Mace, M.J., Fabre-Thorpe, M., 2004. Interaction of top-down and bottom-up processing in the fast visual analysis of natural scenes. Brain Research/Cognitive Brain Research 19 (2), 103-113.

Donchin, E., Coles, M.G.H., 1988. Is the P300 component a manifestation of context updating? Behavioral and Brain Sciences 11, 357-374.
Fabre-Thorpe, M., 2003. Visual categorization: accessing abstraction in non-human primates. Philosophical Transactions: Biological Sciences 358 (1435), 1215-1223.

Fabre-Thorpe, M., Delorme, A., Marlot, C., Thorpe, S., 2001. A limit to the speed of processing in ultra-rapid visual categorization of novel natural scenes. Journal of Cognitive Neuroscience 13 (2), 171-180.

Fillmore, M.T., Vogel-Sprott, M., 2000. Response inhibition under alcohol: effects of cognitive and motivational conflict. Journal of Studies on Alcohol 61 (2), 239-246.

Gratton, G., Coles, M.G., Donchin, E., 1983. A new method for off-line removal of ocular artifact. Electroencephalography and Clinical Neurophysiology 55 (4), 468-484.

Hillyard, S.A., Anllo-Vento, L., 1998. Event-related brain potentials in the study of visual selective attention. Proceedings of the National Academy of Sciences 95 (3), 781-787.

Hurst, P.M., Bagley, S.K., 1972. Acute adaptation to the effects of alcohol. Quarterly Journal of Studies on Alcohol 33 (2), 358-378.

Itti, L., Koch, C., 2001. Computational modelling of visual attention. Nature Review Neuroscience 2 (3), 194-203.

Jääskeläinen, I.P., Näätänen, R., Sillanaukee, P., 1996. Effect of acute ethanol on auditory and visual event-related potentials: a review and reinterpretation. Biological Psychiatry 40 (4), 284-291.

Jääskeläinen, I.P., Hirvonen, J., Kujala, T., Alho, K., Eriksson, C.J.P., Lehtokoski, A., Pekkonen, E., Sinclair, J.D., Yabe, H., Näätänen, R., Sillanaukee, P., 1998. Effects of naltrexone and ethanol on auditory event-related brain potentials. Alcohol 15 (2), 105-111.

Johnson, R., 1988. The amplitude of the P300 component of the eventrelated potentials: review and synthesis. In: Ackles, P., Jennings, J.R., Coles, M.G.H. (Eds.), Advances in Psychophysiology: A Research Annual, vol. 3. JAI Press, Inc., Greenwich, CT, pp. 69-137.

Johnson, R., 1989. Developmental evidence for modality-dependent P300 generators: a normative study. Psychophysiology 26 (6), 651667.

Jones, B.M., 1973. Memory impairment on the ascending and descending limbs of the blood alcohol curve. Journal of Abnormal Psychology 82 (1), 24-32.

Jones, B.M., Vega, A., 1972. Cognitive performance measured on the ascending and descending limb of the blood alcohol curve. Psychopharmacologia 23 (9), 99-114.

Kenemans, J.L., Kok, A., Smulders, F.T., 1993. Event-related potentials to conjunctions of spatial frequency and orientation as a function of stimulus parameters and response requirements. Electroencephalography and Clinical Neurophysiology 88 (1), 51-63.

Kok, A., 2001. On the utility of the P3 amplitude as a measure of processing capacity. Psychophysiology 38 (3), 557-577.

Locke, E.A., Shaw, K.N., Saari, L.M., Latham, G.P., 1981. Goal setting and task performance: 1969-1980. Psychological Bulletin 90 (1), 125-152.

Luck, S.J., Woodman, G.F., Vogel, E.K., 2000. Event-related potential studies of attention. Trends in Cognitive Science 4 (11), 432-440.

Luck, S.J., Hillyard, S.A., 2000. The operation of selective attention at multiple stages of processing. In: Gazzaniga, M.S. (Ed.), The New Cognitive Neurosciences. seconnd ed. MIT Press, Cambridge MA, pp. 687-700.

Makeig, S., Westerfield, M., Jung, T.P., Covington, J., Townsend, J., Sejnowski, T.J., Courchesne, E., 1999. Functionally independent components of the late positive event-related potential during visual spatial attention. Journal of Neuroscience 19 (7), 2665-2680.

Marinkovic, K., Halgren, E., Maltzman, I., 2001. Arousal-related P3a to novel auditory stimuli is abolished by a moderately low alcohol dose. Alcohol 36 (6), 529-539.

Maylor, E.A., Rabbitt, P.M., 1993. Alcohol, reaction time and memory: a meta-analysis. British Journal of Psychology 84 (Pt 3), 301-317.

Michie, P.T., Karayanidis, F., Smith, G.L., Barrett, N.A., Large, M.M., O'Sullivan, B.T., Kavangh, D.J., 1999. An exploration of varieties of visual attention: ERP findings. Cognitive Brain Research 7 (4), 419450 . 
Miller, G.A., Gratton, G., Yee, C.M., 1988. Generalized implementation of an eye movement correction procedure. Psychophysiology 25, 241-243.

Myrsten, A.L., Lamble, R., Frankenhaeuser, M., Lundberg, U., 1979. Interaction of alcohol and reward in an achievement situation. Psychopharmacology (Berlin) 62 (3), 211-215.

Oscar-Berman, M., 1987. Alcohol-related ERP changes in cognition. Alcohol 4 (4), 289-292.

Pashler, H.E., 1999. The Psychology of Attention. MIT Press, Cambridge, MA.

Picton, T.W., Bentin, S., Berg, P., Donchin, E., Hillyard, S.A., Johnson, R., Miller, G.A., Ritter, W., Ruchkin, D.S., Rugg, M.D., Taylor, M.J., 2000. Guidelines for using human event-related potentials to study cognition: Recording standards and publication criteria. Psychophysiology 37, 127152.

Pihl, R.O., Paylan, S.S., Gentes-Hawn, A., Hoaken, P.N.S., 2003. Alcohol affects executive cognitive functioning differentially on the ascending versus descending limb of the blood alcohol concentration curve. Alcoholism: Clinical and Experimental Research 27 (5), 773-779.

Polich, J., Kok, A., 1995. Cognitive and biological determinants of P300: an integrative review. Biological Psychology 41 (2), 103-146.
Potts, G.F., Tucker, D.M., 2001. Frontal evaluation and posterior representation in target detection. Cognitive Brain Research 11 (1), 147-156.

Potts, G.F., 2004. An ERP index of task relevance evaluation of visual stimuli. Brain and Cognition 56 (1), 5-13.

Psychology Software Tools, 1999. E-Prime: Beta 3. Pittsburgh, PA.

Selemon, L.D., Goldman-Rakic, P.S., 1988. Common cortical and subcortical targets of the dorsolateral prefrontal and posterior parietal cortices in the rhesus monkey: evidence for a distributed neural network subserving spatially guided behavior. Journal of Neuroscience 8 (11), 4049-4068.

Steele, C.M., Josephs, R.A., 1988. Drinking your troubles away. II: An attention-allocation model of alcohol's effect on psychological stress. Journal of Abnormal Psychology 97 (2), 196-205.

Thorpe, S., Fize, D., Marlot, C., 1996. Speed of processing in the human visual system. Nature 381 (6582), 520-522.

VanRullen, R., Thorpe, S.J., 2001a. Is it a bird? Is it a plane? Ultra-rapid visual categorisation of natural and artifactual objects. Perception 30 (6), 655-668.

VanRullen, R., Thorpe, S.J., 2001b. The time course of visual processing: from early perception to decision-making. Journal of Cognitive Neuroscience 13 (4), 454-461. 